Alma Mater Studiorum - Università di Bologna DEPARTMENT OF ECONOMICS

\title{
Bertrand versus Cournot with Convex Variable Costs
}

Flavio Delbono

Luca Lambertini

Quaderni - Working Paper DSE N994

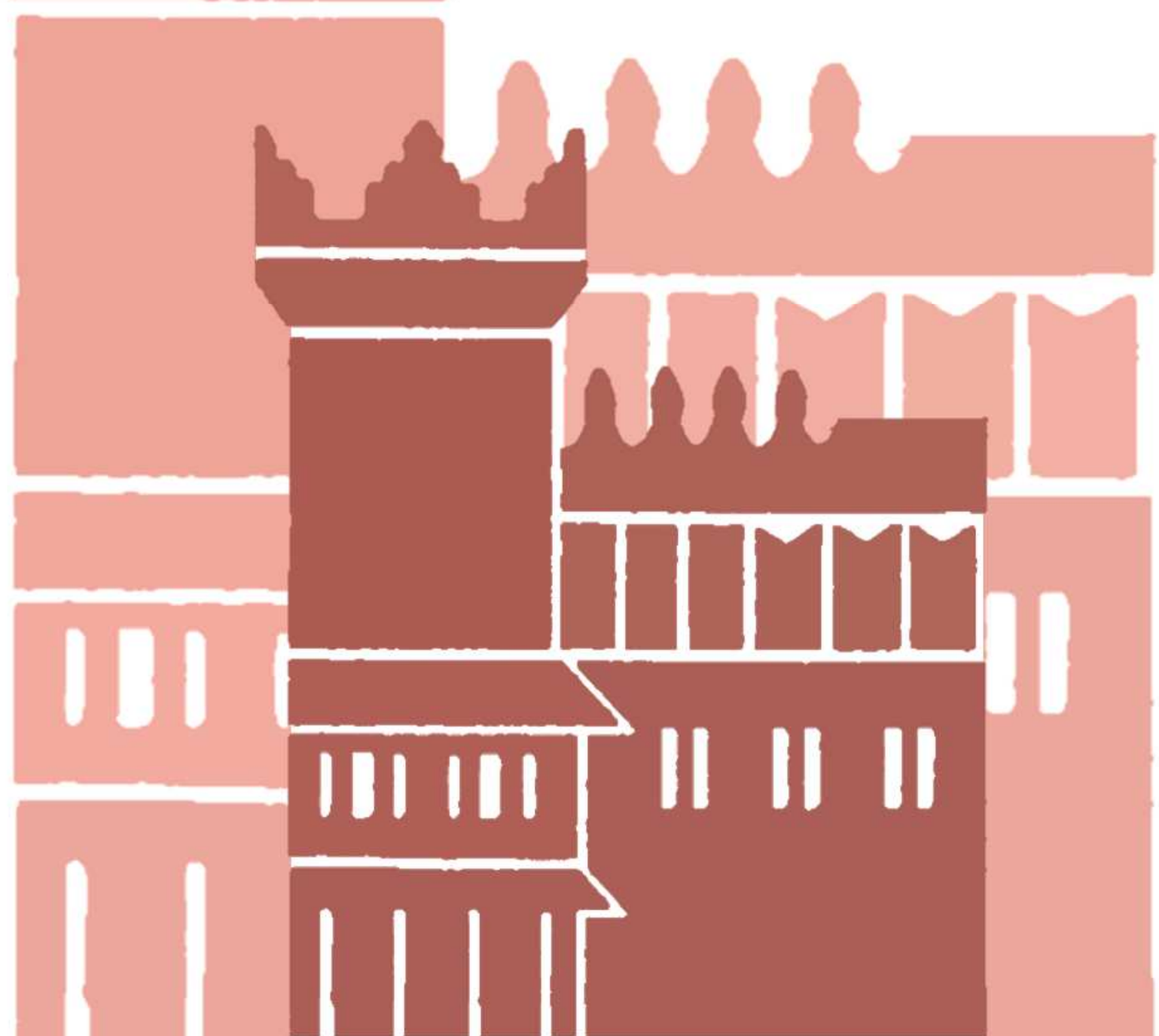




\title{
Bertrand versus Cournot with Convex Variable Costs
}

\author{
Flavio Delbono\# ${ }^{\#}$ and Luca Lambertini ${ }^{\S}$ \\ \# Department of Economics, University of Bologna \\ Piazza Scaravilli 2, 40126 Bologna, Italy \\ flavio.delbono@unibo.it \\ $\S$ Department of Economics, University of Bologna \\ Strada Maggiore 45, 40125 Bologna, Italy \\ luca.lambertini@unibo.it
}

February 26, 2015

\begin{abstract}
Within a simple model of homogeneous oligopoly, we show that the traditional ranking between Bertrand and Cournot equilibria may be reversed. For price setting entails a continuum of price equilibria under convex variable costs, departure from marginal cost pricing may be observed. As a consequence, Bertrand-Nash equilibrium profits (welfare) may be higher (lower) than Cournot-Nash ones. The reversal of the standard rankings occurs when pricing strategies mimic collusive behaviour.
\end{abstract}

JEL Codes: D43, L13

Keywords: oligopoly, pricing strategy, multiple equilibria 


\section{Introduction}

A classical issue in modern industrial organization deals with ranking Nash equilibria generated by price or quantity competition. Absent externalities, the standard conclusion emerging from such comparison states the social superiority of Bertrand competition w.r.t. Cournot competition. This has been proved in a broad class of static games. ${ }^{1}$ However, in a homogeneous product oligopoly, the comparison between the two types of equilibria has been long limited by the strict assumptions about technology needed to ensure the existence of a pure strategy equilibrium under Bertrand rules. Such a limitation has been bypassed by Dastidar (1995), proving that, under concave demand and convex costs, price competition in a homogeneous oligopoly yields a continuum of Bertrand-Nash equilibria in pure strategies. This result may then allow one to challenge the alleged greater efficiency of Bertrand-Nash equilibria w.r.t. the Cournot-Nash equilibrium. As long as Bertrand-Nash behaviour doesn't need to coincide with marginal cost pricing, the standard ranking between Bertrand-Nash and Cournot-Nash profits and social welfare may be reversed. In this note, indeed, we show that, in the continuum of price equilibria under convex variable costs, departure from marginal cost pricing may be observed. As a result, in a broad range of the parameter constellation, Bertrand-Nash equilibrium profits (welfare) may be higher (lower) than Cournot-Nash ones. It's worth noting that the reversal of the standard rankings occurs when pricing strategies mimic collusive behaviour.

The remainder of the paper is organised as follows. In section 2 we set up the model and solve the two games. In section 3, we perform some comparative statics, instrumental to our main results illustrated in section 4. Section 5 concludes.

\footnotetext{
${ }^{1}$ See, fon instance, Singh and Vives (1984), Vives (1985), Okuguchi (1987) and Dastidar (1997). With cost asymmetry and a small degree of product differentiation, Zanchettin (2006) shows that the opposite can occur.
} 


\section{Setup and Nash equilibria}

Consider a market supplied by a set $\mathcal{N}=1,2,3, \ldots, n$ of identical firms producing a homogeneous good whose demand function is $p=1-Q$, where $Q=\sum_{i=1}^{n} q_{i}$ is aggregate output and $p$ is price. All firms share the same technology, summarised by the convex cost function $C_{i}=c q_{i}^{2} / 2$. Accordingly, the profit function of firm $i$ is

$$
\pi_{i}=\left(p-\frac{c q_{i}}{2}\right) q_{i}=\left(1-q_{i}-Q_{-i}-\frac{c q_{i}}{2}\right) q_{i}
$$

where $Q_{-i}=\Sigma_{j \neq i} q_{j}$.

Firms play simultaneously a non-cooperative one-shot game under complete, symmetric and imperfect information. The solution concept is the Nash equilibrium.

\subsection{The quantity-setting game}

If firms are Cournot players, the relevant first order condition for firm $i$ is:

$$
\frac{\partial \pi_{i}}{\partial q_{i}}=1-2 q_{i}-Q_{-i}-c q_{i}=0
$$

which, under the symmetry condition $q_{j}=q_{i}=q$ for all $i$ and $j$, yields the Cournot-Nash $(C N)$ equilibrium output

$$
q^{C N}=\frac{1}{n+1+c}
$$

for each individual firm. The resulting equilibrium profits are

$$
\pi^{C N}=\frac{2+c}{2(n+1+c)^{2}}
$$

and social welfare is

$$
S W^{C N}=n \pi^{C N}+C S^{C N}=\frac{n(n+2+c)}{2(n+1+c)^{2}}
$$

where $C S^{C N}=\left(n q^{C N}\right)^{2} / 2$ is consumer surplus. 


\subsection{The price-setting game}

Here, we follow Dastidar (1995), where it is shown that, if costs are strictly convex in output levels, Bertrand competition yields a continuum of Nash equilibria. The Nash equilibrium in pure strategies involves indeed all firms setting the same price $p^{*} \in\left[p^{a v c}, p^{u}\right]$. At the lower bound $p^{a v c}$, equilibrium price equals average variable costs, so that firms would be indifferent between producing or not. At the upper bound $p^{u}$, the equilibrium price is such that firms would be indifferent between playing $p^{u}$ or marginally undercutting it in order to capture the entire market demand.

The range of equilibrium prices is identified by: ${ }^{2}$

$$
p^{B N}=\frac{c}{c+2(n-\alpha)}
$$

where $B N$ mnemonics for Bertrand-Nash, and $\alpha$ is a non-negative parameter whose range, to be specified below, determines the continuum of equilibrium prices. The associated individual output and profits are

$$
\begin{aligned}
q^{B N} & =\frac{2(n-\alpha)}{n[c+2(n-\alpha)]} \\
\pi^{B N} & =\frac{2 \alpha c(n-\alpha)}{n^{2}[c+2(n-\alpha)]^{2}}
\end{aligned}
$$

and social welfare is

$$
S W^{B N}=n \pi^{B N}+C S^{B N}=\frac{2(n-\alpha)[n(n-\alpha)+c \alpha]}{n[2(n-\alpha)+c]^{2}}
$$

The admissible range is $\alpha \in\left[0, n^{2} /(1+n)\right]$. This is because

- in $\alpha=0$, the equilibrium price equals average variable cost;

- at $\alpha=n / 2$, marginal cost pricing obtains;

- if $\alpha=n^{2} /(1+n), p^{B N}$ reaches the highest level above which undercutting takes place.

\footnotetext{
${ }^{2}$ See Dastidar (1995, pp. 27-28); and Gori et al. (2014, pp. 373-75).
} 


\section{Comparative statics}

The very fact of the existence of a continuum of price equilibria ranging well above marginal cost pricing raises two related questions. The first deals with the monotonicity (or the lack thereof) of equilibrium profits w.r.t. the number of firms under Bertrand competition. The second issue is whether the profit ranking across the two regimes is robust to variations in industry structure as measured by the number of firms, and/or the price mark-up determined by the value of $\alpha$. In this section we tackle the first question, while the second is postponed to the next section.

For completeness, we set out by summarising the effect of an increase in $n$ on Cournot-Nash equilibrium profits. This is captured by the following derivative: ${ }^{3}$

$$
\frac{\partial \pi^{C N}}{\partial n}=-\frac{c+2}{(n+1+c)^{3}}<0
$$

everywhere. This is the standard result we are well accustomed with, telling that individual profits are monotonically decreasing in the number of quantitysetting firms.

Now we examine the behaviour of Bertrand profits w.r.t. $n$ in our setting, where there exists a continuum of equilibria. We are going to prove the following:

Lemma $1 \partial \pi^{B N} / \partial n>0$ for all $n \in\left(n_{-}^{B}, n_{+}^{B}\right)$ and negative elsewhere, with

$$
n_{ \pm}^{B}=\frac{10 \alpha-c \pm \sqrt{c^{2}+28 c \alpha+4 \alpha^{2}}}{12}
$$

Proof. The partial derivative of Bertrand-Nash profits w.r.t. $n$ is:

$$
\frac{\partial \pi^{B N}}{\partial n}=\frac{2 c \alpha\left[2 \alpha(5 n-2 \alpha)-6 n^{2}-c(n-2 \alpha)\right]}{n^{3}[c+2(n-\alpha)]^{3}}
$$

\footnotetext{
${ }^{3}$ As usual, we are treating $n$ as a continuous magnitude when performing comparative statics. As soon as we will be looking at numerical examples, we will confine our attention to integers.
} 
Since $n>n^{2} /(n+1)$, which is the upper bound of the admissible interval for $\alpha$, the denominator of (11) is strictly positive. Hence, the sign of $\partial \pi^{B N} / \partial n$ is the sign of the numerator. The roots of

$$
2 \alpha(5 n-2 \alpha)-6 n^{2}-c(n-2 \alpha)=0
$$

are

$$
n_{ \pm}^{B}=\frac{10 \alpha-c \pm \sqrt{c^{2}+28 c \alpha+4 \alpha^{2}}}{12}
$$

and, given the concavity of the numerator w.r.t. $n$, this implies that $\partial \pi^{B N} / \partial n>$ 0 for all $n \in\left(n_{-}^{B}, n_{+}^{B}\right)$. Outside this range, $\partial \pi^{B N} / \partial n<0$.

Notice that, in order for the interval $\left(n_{-}^{B}, n_{+}^{B}\right)$ to be economically meaningful, it must be that at least $n_{+}^{B} \geq 2$, i.e.,

$$
n_{+}^{B}-2=\frac{10 \alpha-24-c+\sqrt{c^{2}+28 c \alpha+4 \alpha^{2}}}{12} \geq 0
$$

The existence of a range of industry structures wherein an increase in the number of firms yields an increase in the Bertrand-Nash profits suggests that Bertand-Nash profits might overcome those generated by those associated to the Cournot-Nash equilibrium. In the next section we show that this can indeed happen in an admissible portion of the parameter space.

\section{Ranking equilibrium profits and welfare}

Under marginal cost pricing, it would be true that $\pi^{C N}>\pi^{B N}$ for all $n \geq$ 2. However, since we follow Dastidar's (1995) approach to model Bertrand competition, we have to admit the possibility for $\pi^{B N}$ to increase in $n$ due to the presence of a mark-up exceeding its competitive level as $\alpha$ increases above $n / 2$.

To investigate whether this brings about a reversal of fortune across equilibria, it is appropriate to redefine the upper bound of $\alpha$ in terms of a lower 
bound to $n$. This trivially requires solving the following inequality:

$$
\alpha(n+1) \leq n^{2}
$$

w.r.t. $n$, which delivers the equivalent condition

$$
n \geq \frac{\alpha+\sqrt{\alpha(\alpha+4)}}{2} \equiv \underline{n}
$$

If one compares $\underline{n}$ against $n_{ \pm}^{B}$, it turns out that

$$
\underline{n}-n_{-}^{B}=\frac{c-4 \alpha+6 \sqrt{\alpha(\alpha+4)}+\sqrt{c^{2}+28 c \alpha+4 \alpha^{2}}}{12}>0
$$

everywhere, because $3 \sqrt{\alpha(\alpha+4)}>2 \alpha$. Therefore, $\underline{n}>n_{-}^{B}$ always.

The comparison between $\underline{n}$ and $n_{ \pm}^{B}$ involves evaluating the sign of the following expression:

$$
\underline{n}-n_{+}^{B}=\frac{c-4 \alpha+6 \sqrt{\alpha(\alpha+4)}-\sqrt{c^{2}+28 c \alpha+4 \alpha^{2}}}{12}
$$

Again, $c-4 \alpha+6 \sqrt{\alpha(\alpha+4)}>0$ since $3 \sqrt{\alpha(\alpha+4)}>2 \alpha$. Consequently, the sign of $\underline{n}-n_{+}^{B}$ is the sign of

$$
[c-4 \alpha+6 \sqrt{\alpha(\alpha+4)}]^{2}-\left(c^{2}+28 c \alpha+4 \alpha^{2}\right)
$$

which can be usefully rewritten as

$$
48 \alpha(3+\alpha-\sqrt{\alpha(\alpha+4)})+12 c(\sqrt{\alpha(\alpha+4)}-3 \alpha)
$$

where $48 \alpha(3+\alpha-\sqrt{\alpha(\alpha+4)})>0$ for all admissible values of $\alpha$, while $\sqrt{\alpha(\alpha+4)}-3 \alpha \gtreqless 0$ for all $\alpha \lesseqgtr 1 / 2$. Thus,

- if $\alpha \in[0,1 / 2], \underline{n}>n_{+}^{B}$ everywhere;

- if $\alpha>1 / 2$, (i) $\underline{n}>n_{+}^{B}$ for all $c \in(0, \widetilde{c})$; (ii) $\underline{n} \leq n_{+}^{B}$ for all $c \geq \widetilde{c}$, with

$$
\widetilde{c} \equiv \frac{\alpha(2 \alpha+5)-(2 \alpha-3) \sqrt{\alpha(\alpha+4)}}{2 \alpha-1} .
$$


When $\alpha>1 / 2, \partial \widetilde{c} / \partial \alpha<0$ and $\partial^{2} \widetilde{c} / \partial \alpha^{2}>0$, as illustrated in Figure 1 .

Figure 1 The critical theshold of $c$.

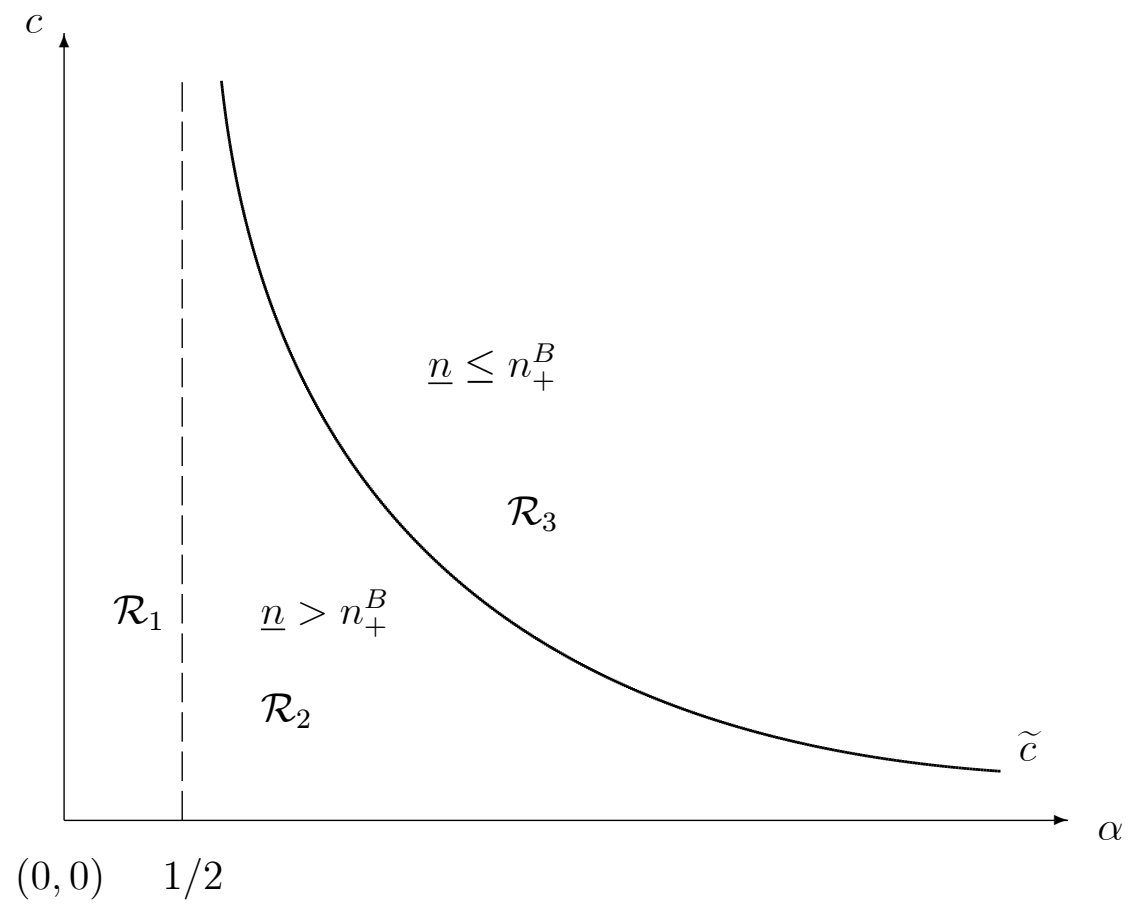

Accordingly, we may identify three regions: $\mathcal{R}_{1} \equiv\{\alpha \in[0,1 / 2], c>0\}$; $\mathcal{R}_{2} \equiv\{\alpha>1 / 2, c \in(0, \widetilde{c})\} ; \mathcal{R}_{3} \equiv\{\alpha>1 / 2, c>\widetilde{c}\}$. By inspecting the ranking between $\underline{n}$ and $n_{+}^{B}$ in these three regions, we draw the following:

Lemma 2 In $\widehat{\mathcal{R}} \equiv \mathcal{R}_{1} \cup \mathcal{R}_{2}, \underline{n}>n_{+}^{B}$. In $\mathcal{R}_{3}, \underline{n} \leq n_{+}^{B}$.

In the space $(c, n)$, Lemma 2 gives rise to Figures 2-3, where $\underline{n}$ is a flat line because it is independent of $c$. In Figure $2, \alpha \in[0,1 / 2]$, so that $\underline{n}>n_{+}^{B}>n_{-}^{B}$. Since $n$ must be at least as high as $\underline{n}$, in this case $\partial \pi^{B N} / \partial n<0$ for all admissible $n$. 
Figure 2 The sign of $\partial \pi^{B N} / \partial n$ in the space $(c, n), \alpha<1 / 2$.

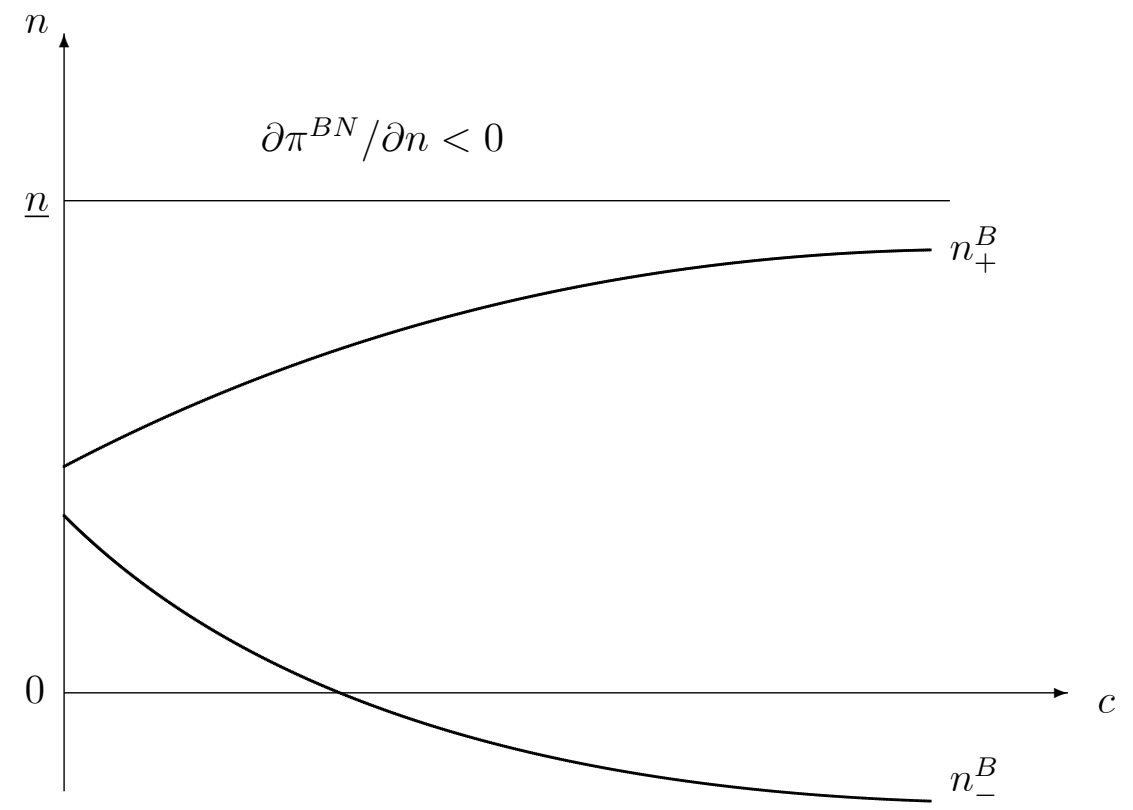

In Figure 3, $\alpha>1 / 2$ and therefore $\underline{n} \gtreqless n_{+}^{B}$ for all $c \lesseqgtr \widetilde{c}$, and $\underline{n}, n_{+}^{B}>n_{-}^{B}$ for all $c>0$. In this case, as soon as $n_{+}^{B}>\underline{n}$, we have a region wherein $\partial \pi^{B N} / \partial n>0$ 
Figure 3 The sign of $\partial \pi^{B N} / \partial n$ in the space $(c, n), \alpha>1 / 2$.

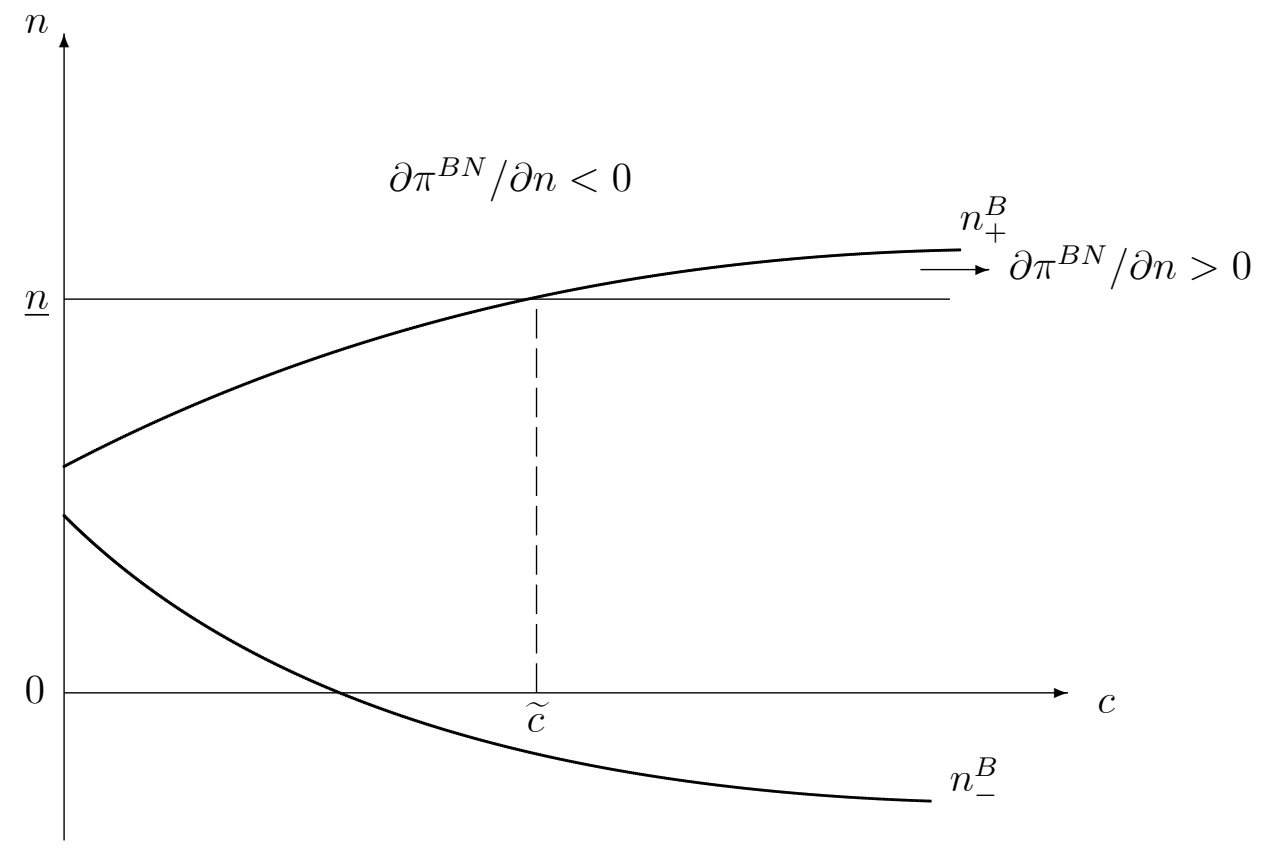

Since it must be $n \geq \underline{n}$, then Lemma 2 delivers, without further proof, the following result:

Proposition 3 If $\alpha \in[0,1 / 2], \partial \pi^{B N} / \partial n<0$ for all $c>0$ and all $n \geq \underline{n}$. If $\alpha>1 / 2, \partial \pi^{B N} / \partial n>0$ for all $c>\widetilde{c}$ and all $n \in\left(\underline{n}, n_{+}^{B}\right)$, while it is negative in the remainder of the admissible parameter range.

Now we are ready to characterise the ranking of equilibrium profits and welfare between Bertrand and Cournot equilibria. To perform this task, we produce the necessary and sufficient condition for $\pi^{B N}>\pi^{C N}$ : 
Proposition 4 Take $\alpha \in\left(\frac{n}{2}, \frac{n^{2}}{n+1}\right]$. Define

$$
\begin{gathered}
c_{1} \equiv \frac{2(n-\alpha)}{2 \alpha-n} \\
c_{2} \equiv \frac{2 n(n-\alpha)-\alpha+\sqrt{\alpha\left[4 n^{3}+\alpha+4 n(\alpha-n(\alpha+1))\right]}}{2 \alpha-n}
\end{gathered}
$$

For all $c \in\left(\min \left\{\widetilde{c}, c_{1}\right\}, c_{2}\right), \pi^{B N}>\pi^{C N}$ and $S W^{C N}>S W^{B N}$.

Proof. The difference between $\pi^{B N}$ and $\pi^{C N}$ can be written as $\pi^{B N}-\pi^{C N}=\frac{[c(2 \alpha-n)-2(n-\alpha)]\left[4 n^{2}(n-\alpha)+2 c(2 n(n-\alpha)-\alpha)-c^{2}(2 \alpha-n)\right]}{2 n^{2}(n+1+c)^{2}[2(n-\alpha)+c]^{2}}$

The denominator of the r.h.s. of (22) is always positive. The numerator is nil at

$$
\begin{gathered}
c_{1} \equiv \frac{2(n-\alpha)}{2 \alpha-n} \\
c_{2} \equiv \frac{2 n(n-\alpha)-\alpha+\sqrt{\alpha\left[4 n^{3}+\alpha+4 n(\alpha-n(\alpha+1))\right]}}{2 \alpha-n} \\
c_{3} \equiv \frac{2 n(n-\alpha)-\alpha-\sqrt{\alpha\left[4 n^{3}+\alpha+4 n(\alpha-n(\alpha+1))\right]}}{2 \alpha-n}
\end{gathered}
$$

with $c_{2}>c_{1}>c_{3}$ for all $\alpha \in\left(\frac{n}{2}, \frac{n^{2}}{n+1}\right]$ and $n \geq 2$.

Given that $c(2 \alpha-n)-2(n-\alpha)>0$ for all $c>c_{1}$ and

$$
4 n^{2}(n-\alpha)+2 c(2 n(n-\alpha)-\alpha)-c^{2}(2 \alpha-n)>0
$$

for all $c \in\left(c_{3}, c_{2}\right), \pi^{B N}>\pi^{C N}$ for all $c \in\left(0, c_{3}\right)$ and all $c \in\left(c_{1}, c_{2}\right)$. However, from Proposition 3, we have to account for the lower bound to $c$ for $\partial \pi^{B N} / \partial n>0$, i.e., $c>\widetilde{c}$, with $\widetilde{c} \in\left(c_{3}, c_{2}\right)$ everywhere, although $\widetilde{c}$ may be 
higher or lower than $c_{1}$ in the admissible range of $n$ and $\alpha$. Hence, we may disregard all $c \in\left(0, c_{3}\right)$, and we are left with the interval $c \in\left(\min \left\{\widetilde{c}, c_{1}\right\}, c_{2}\right)$, where indeed $\pi^{B N}>\pi^{C N}$ holds.

The difference between welfare levels writes:

$S W^{C N}-S W^{B N}=\frac{[2(n-\alpha)-c(2 \alpha-n)]\left[c\left(n^{2}-2 \alpha(n+1)\right)-2 n(n-\alpha)-c^{2}(2 \alpha-n)\right]}{2 n(n+1+c)^{2}[2(n-\alpha)+c]^{2}}$

Solving $S W^{C N}-S W^{B N}=0$ w.r.t. $c$, one obtains the following roots:

$$
\begin{gathered}
c_{1} \equiv \frac{2(n-\alpha)}{2 \alpha-n} \\
c_{4} \equiv \frac{n(n-2 \alpha)-2 \alpha+\sqrt{8(n-2 \alpha)(n-\alpha)+[n(n-2 \alpha)-2 \alpha]^{2}}}{2(2 \alpha-n)} \\
c_{5} \equiv \frac{n(n-2 \alpha)-2 \alpha-\sqrt{8(n-2 \alpha)(n-\alpha)+[n(n-2 \alpha)-2 \alpha]^{2}}}{2(2 \alpha-n)}
\end{gathered}
$$

Notice that solution $c_{1}$ is the same root solving $\pi^{C N}=\pi^{B N} .{ }^{4}$ Moreover, the remaining two roots might not be real because $\alpha>n / 2$ as price becomes larger than marginal cost in the Bertrand case. If $c_{4}, c_{5} \in \mathbb{R}$, then $c_{1}>c_{4}>$ $c_{5}$.Then, observe that $c_{4}$ and $c_{5}$ are generated by the expression appearing in the second square bracket at the numerator of (25), which is parabolic and concave in $c$. Therefore, two cases may arise:

- $c_{4}, c_{5} \in \mathbb{R}$ : if so, then $c_{1}>c_{4}>c_{5}$ and consequently $S W^{C N}>S W^{B N}$ for all $c>c_{1}$;

- $c_{4}, c_{5} \notin \mathbb{R}$ : if so, then $c\left(n^{2}-2 \alpha(n+)\right)-2 n(n-\alpha)-c^{2}(2 \alpha-n)<0$ because the coefficient of $c^{2}$ is negative above marginal cost pricing. Hence, again, $S W^{C N}>S W^{B N}$ for all $c>c_{1}$.

\footnotetext{
${ }^{4}$ Incidentally, it is worth noting that $c_{1}$ also solves $p^{C N}=p^{B N}$, with $p^{C N}<p^{B N}$ for all $c>c_{1}$. By the same token, $q^{C N}>q^{B N}$ for all $c>c_{1}$.
} 
This completes the proof.

The intuition behind Proposition 4 can be explained as follows. First, notice that the Bertrand-Nash equilibrium profits outperform profits in the Cournot setting when $\partial \pi^{B N} / \partial n>0$, as from Proposition 3. This anticompetitive effect of expanding the population of firms on Bertrand profits occurs when both $\alpha$ and the parameter scaling marginal cost, $c$, are sufficiently high. As one can see from (6), $p^{B N}$ is increasing in both parameters. Hence, $\partial \pi^{B N} / \partial n>0$ when the Bertrand equilibrium price significantly departs from marginal cost pricing to mimic collusive behaviour. Another way of grasping the result consists in observing that, in the region $\alpha \in\left(n / 2, n^{2} /(n+1)\right], p^{B N}>p^{C N}$ and $q^{C N}<q^{B N}$ for all $c>c_{1}$, which amounts to saying that if the Bertrand equilibrium price departs from marginal cost and the latter is sufficiently high, then the Cournot output becomes lower than the Bertrand one, causing the profit and welfare ranking to flip over.

\subsection{An example}

In order to construct an example where $\partial \pi^{B N} / \partial n>0, \pi^{B N}>\pi^{C N}$ and $S W^{C N}>S W^{B N}$, we have to take a triple $\{\alpha, c, n\}$ satisfying all of the aforementioned constraints, i.e.:

- $\alpha \in\left(\frac{n}{2}, \frac{n^{2}}{n+1}\right]$

- $c \in\left(\min \left\{\widetilde{c}, c_{1}\right\}, c_{2}\right)$

- $n \in\left(\min \{2, \underline{n}\}, n_{+}^{B}\right)$

One such triple is $\{\alpha=4, c=6, n=5\}$, at which

$\frac{\partial \pi^{B N}}{\partial n}=\frac{3}{1000} ; \pi^{B N}=\frac{3}{100} ; \pi^{C N}=\frac{1}{36} ; S W^{C N}=\frac{65}{288} ; S W^{B N}=\frac{29}{160}$ 


$$
\begin{gathered}
\pi^{B N}-\pi^{C N}=\frac{1}{450} ; S W^{C N}-S W^{B N}=\frac{2}{45} \\
\widetilde{c}=\frac{4(13-5 \sqrt{2})}{7}=3.38 ; c_{1}=\frac{2}{3} ; c_{2}=2\left(1+2 \sqrt{\frac{7}{3}}\right)=8.11 \\
n_{+}^{B}=\frac{17+\sqrt{193}}{6}=5.14 ; \underline{n}=2(1+\sqrt{2})=4.83
\end{gathered}
$$

\section{Concluding remarks}

In this paper we have proved that the traditional ranking between Bertrand and Cournot equilibria may be reversed under convex variable costs. The presence of a continuum of price equilibria allows for departures from marginal cost pricing, to such an extent that Bertrand-Nash equilibrium profits become higher than Cournot-Nash ones. In the same region of parameters where this happens, the opposite occurs to the sequence of social welfare levels. These reversals are driven by the fact that equilibrium pricing mimics collusion. 


\section{References}

[1] Dastidar, K.G. (1995), "On the Existence of Pure Strategy Bertrand Equilibrium", Economic Theory, 5, 9-32.

[2] Dastidar, K.G. (1997), "Comparing Cournot and Bertrand in a Homogeneous Product Market", Journal of Economic Theory, 75, 205-12.

[3] Gori, G., L. Lambertini and A. Tampieri (2014), "Trade Costs, FDI Incentives, and the Intensity of Price Competition", International Journal of Economic Theory, 10, 371-85.

[4] Okuguchi, K. (1987), "Equilibrium Prices in the Bertrand and Cournot Oligopolies", Journal of Economic Theory, 42, 128-39.

[5] Singh, N. and X. Vives (1984), "Price and Quantity Competition in a Differentiated Duopoly", RAND Journal of Economics, 15, 546-54.

[6] Vives, X. (1985), "On the Efficiency of Bertrand and Cournot Equilibria with Product Differentiation", Journal of Economic Theory, 36, 166-75.

[7] Zanchettin, P. (2006), "Differentiated Duopoly with Asymmetric Costs", Journal of Economics and Management Strategy, 15, 999-1015. 


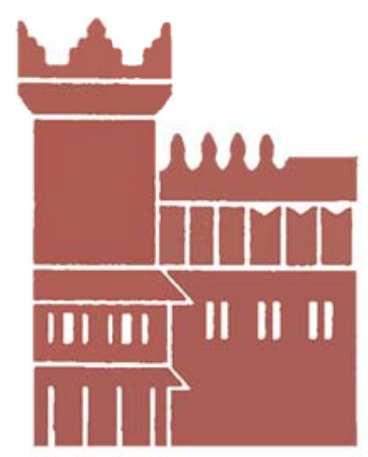

Alma Mater Studiorum - Università di Bologna DEPARTMENT OF ECONOMICS

Strada Maggiore 45

40125 Bologna - Italy

Tel. +39051 2092604

Fax +390512092664

http://www.dse.unibo.it 